\title{
Biocatalytic Synthesis of Angiotensin-converting Enzyme (ACE) Inhibitor Drug Precursors Using Membrane Bioreactor: A Review
}

\author{
A. L. Ahmad ${ }^{1 *}$, P. C. $\mathrm{Oh}^{2} \&$ S. R. Abd. Shukor ${ }^{3}$ \\ ${ }^{1,2 \& 3}$ School of Chemical Engineering, Engineering Campus, Universiti Sains Malaysia, 14300 Nibong Tebal, \\ Penang, Malaysia
}

\begin{abstract}
Over the past decade, the production of angiotensin-converting enzyme (ACE) inhibitor drug intermediates has become increasingly important in the pharmaceutical industry. The most significant clinical application of ACE inhibitors is in the management of hypertension and cardiovascular diseases, particularly congestive heart failure (CHF). A number of chemical methods have been reported thus far for the synthesis of ACE inhibitor precursors. However, chemical methods generally suffer from process complexity, high cost, and environmental pollution. On the other hand, enantiomerically pure ACE inhibitor drug precursors can be obtained elegantly and efficiently by employing biocatalytic methods, where it appears to be the most at tractive process in terms of potential industrial applications and green chemistry. In the recent past, membranes are expeditiously used in the enzyme-catalyzed synthesis of optically active pharmaceutical compounds on laboratory and industrial scales, being incorporated in membrane bioreactor system. This technique can be applied for the repeated use of the enzymes in the synthesis via retention or immobilization, especially when dealing with costly biocatalysts. Herein we review the enzymatic synthesis of vital ACE inhibitor drug precursors as potentially useful intermediates in the synthesis of pharmaceutical products, in environmentally friendly condition, using membrane bioreactor for biotransformation.
\end{abstract}

Keywords: Membrane bioreactor, ACE inhibitors, drug precursors, enzyme-catalyzed processes, pharmaceuticals

\subsection{INTRODUCTION}

Over the last 20 years, angiotensin-converting enzyme (ACE) inhibitors have achieved widespread usage as one of the first line drugs for treatment of hypertension and congestive heart failure [1-3]. ACE inhibitors have long been the cornerstone for therapy of cardiovascular disorders, chronic heart failure, hypertension, left ventricular dysfunction as well as prevention of nephropathy in diabetes mellitus. Recent clinical trials have shown that ACE inhibitors are superior to other classes of drugs in the reduction of morbidity and mortality [4], hence establishing its importance in the pharmaceutical industry. Virtually all ACE inhibitors with therapeutic

" Corresponding author: A. L. Ahmad (email: chlatif@eng.usm. my) significance such as enalapril, delapril, lisinopril, quinapril, ramipril, trandolapril, cilazapril and benzapril (Figure 1) $[5,6]$, refer to (S)-2-amino4-phenylbutanoic acid and (R)-2-hydroxy-4phenylbutanoic acid or their esters as a common building block, due to the presence of (S)homophenylalanine moiety as the central pharmacophore unit [7].

Thus far, there exist many conceivable methods of producing enantiomerically pure ACE inhibitor drug precursors, more commonly via chemical $[8,9]$, microbial or enzymatic routes by way of kinetic resolution or asymmetric synthesis. Chemical methods generally suffer from process complexity, high cost, and environmental pollution. The advantages of microbial or enzyme-catalyzed reactions over chemical synthesis are that reactions involving biocatalysts are often highly stereo- 
<smiles>O=C(O)[C@H](O)CCc1ccccc1</smiles>

(R)-2-hydroxy-4-phenylbutanoic acid

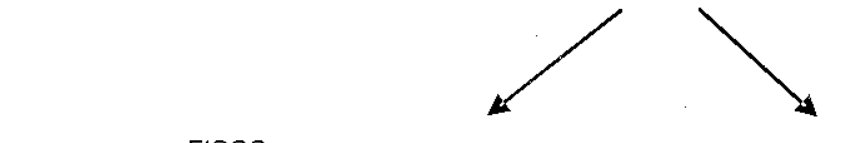<smiles>CCOC(=O)[C@H](CCc1ccccc1)N[C@@H](C)C(=O)N1CCC[C@H]1C(=O)O</smiles>

Enalapril<smiles>NCCCC[C@H](N[C@@H](CCc1ccccc1)C(=O)O)C(=O)N1CCC[C@H]1C(=O)O</smiles>

Lisinopril<smiles>[R]OC(=O)[C@H](C)N[C@@H](CCc1ccccc1)C(=O)N1C[C@H]2CCC[C@H]2[C@H]1C(=O)O</smiles><smiles>O=C(O)[C@H](CCc1ccccc1)NC1CCCN2CCC[C@H](C(=O)O)N1C2=O</smiles><smiles>CCOC(C)N[C@@H](CCc1ccccc1)C(=O)N(CC(=O)O)C1Cc2ccccc2C1</smiles><smiles>CCOC(CCc1ccccc1)N[C@H](C)C(=O)N1Cc2ccccc2C[C@H]1C(=O)O</smiles><smiles>COC(=O)[C@H](CCc1ccccc1)N[C@@H](C)C(=O)N1C[C@H]2CCCC[C@H]2[C@H]1C(=O)O</smiles><smiles>CCOC(=O)[C@H](CCc1ccccc1)N[C@H]1CCc2ccccc2N(CC(=O)O)C1=O</smiles>

Figure 1 ACE inhibitors with (S)-homophenylalanine moiety

selective [10]. They can be carried out at ambient temperature and atmospheric pressure, avoiding the use of more extreme conditions, therefore minimizing problems of isomerization, race- mization, epimerization or rearrangement [11]. Ribeiro and coworkers [12-15] have successfully rationalized the vantage of biocatalytic processes, which are generally carried out in aqueous solvent, 
avoiding the usage of environmentally hamful chemicals and solvent waste disposition. Biocatalysts used can be retained and reused in many cycles. It is thence worth nothing that biocatalytic synthesis of chiral building blocks appears to be the more attractive process in terms of potential industrial applications and green chemistry, despite the fact that conventional chemical processes lead to satisfactory yields and enantiomeric excesses.

Enantiopure ACE inhibitor intermediates could be obtained by way of racemate resolution or asymmetric synthesis. Methods for racemate resolution include direct resolution by entrainment, crystallization of diastereoisomeric salts [16] and kinetic resolution [17], with a maximum theoretical yield of $50 \%$ [18]. Asymmetric synthesis involves the enantioselective conversion of prochiral substrates to an optically active product, contributing to a theoretical yield of $100 \%$. Thus, a cursory appraisal of the relative economics racemate resolution versus asymmetric synthesis indicates a preference for the latter since it has a theoretical yield of $100 \%$. Besides that, racemate resolution also has a major setback as it requires at least one extra step to racemize the unwanted isomer for production of enantiopure intermediates $[7,19,20]$. Although various solutions were developed in the scientific community to overcome this drawback including dynamic resolution by in situ racemization [21], the process often incur extra cost, thus further favoring asymmetric synthesis which are normally applied for pharmaceuticals synthesis using membrane bioreactors.

\subsection{BIOTRANSFORMATION FOR ACE INHIBITOR DRUG INTERMEDIATE PRODUCTION}

Relationships between, and co-existence of, membranes and biocatalysts is ubiquitous throughout nature. Thus, before we delve into the production of ACE inhibitor drug precursors using a membrane bioreactor, herein we provide a prefatory review of the biocatalytic procedures generally used for their synthesis. Asymmetric reduction of prochiral ketone remains one of the most investigated methods to date for production of chiral ACE inhibitor intermediates, as mentioned in the previous section. Many successful advances have been published thus far, with reproducible results of satisfactory yield. One of the most established method for synthesizing ACE inhibitor drug precursor, namely (S)-2amino-4-phenylbutanoic acid and (R)-2-hydroxy4-phenylbutanoic acid on a laboratory scale was carried out via enzyme-catalyzed asymmetric synthesis of keto acids [22, 23]. In this method, prochiral ketone was converted via reductive amination to enantiopure products [24] with bulky side chains by addition of biocatalysts such as Lhomophenylalanine dehydrogenase [25] or Dlactate dehydrogenase in the presence of cofactor. Since the coenzyme is expensive, an efficient and cost-effective in situ regeneration of the compound is prerequisite in order to meet the economic constraints [26]. For this reaction, NADH was regenerated in parallel using formate dehydrogenase which catalyzes the oxidation of formate to $\mathrm{CO}_{2}$ with the concomitant reduction of NAD to NADH [27-29]. The equilibrium of the reaction is very favorable as the byproduct $\mathrm{CO}_{2}$ which is not obnoxious to the drug precursors can be easily remioved. The equilibrium of the enzyme-catalyzed reaction with cofactor regeneration strongly favors the ACE inhibitor intermediate synthesis therefore high conversion could be obtained, especially if a membrane bioreactor module is employed as will be discussed in the subsequent section.

Another enzyme-catalyzed method involves the facile synthesis of (S)-2-amino-4-phenylbutanoic acid, an ACE inhibitor intermediate, which was successfully accomplished with sufficient yield by Chen and colleagues [30]. In this work, genetically engineered tyrosine-aminotransferase-catalyzed synthesis was carried out for production of drug intermediate. Genetically engineered tyrosine aminotransferase is produced by overexpressing the gene product in Escherichia Coli, and generally has an enzymatic activity of approximately 109.8 unit/ $\mathrm{ml}$ in the culture media. It is noted that any commercially produced genetically engineered tyrosine aminotransferase are equally applicable. (S)-2-amino-4-phenylbutanoic acid which precipitates easily from the solution under reaction conditions could be produced in high yield and enantiomeric excess in a laboratory-scaled unit. 
Besides that, (S)-2-amino-4-phenylbutanoic acid was also produced by using microbial cells containing aminotransferase activity as reported by Senuma, Cho and coworkers $[31,32]$. Senuma and coauthors successfully prepared ACE inhibitor drug precursor with high yield using Paracoccus denitrificans. Cho and collegues also synthesized the compound asymmetrically using a recombinant aromatic amino acid transaminase in the reaction media which permits efficient synthesis of ACE intermediates using a single transaminase reaction. Nevertheless, the aminotransferase activity is markedly inhibited by a high concentration of substrate in the reaction mixture leading to limitations in large-scale production.

Hydantoinase processes have newly been established by Kao [33] for production of (S)-2amino-4-phenylbutanoic acid using recombinant Escherichia coli cells with dihydropyrimidinase and L-N-carbamoylase activities as whole cell biocatalysts which are thermostable for a substantial duration under the reaction conditions used. However, further study is needed to develop the desired enzyme with substrate preference to the (S) enantiomer in order to improve the achieved yield.

In a research work published by de Lacerda and colleague $[12,34]$, potential tools for the desired biotransformation to acquire (R)-2hydroxy-4-phenylbutyrate, a key intermediate in the production of ACE inhibitors was investigated. Various microorganisms including Kluyveromyces marxianus, Pichia pastoris, Pichia anomala and Pichia angusta were used in the experiment, where high conversion and enantioselectivity was ultimately obtained by utilization of $P$. angusta. The product was synthesized via microbial reduction in an aqueous medium, thus established an attractive process in terms of potential industrial application and green chemistry.

Alternatively, immobilized whole cells of Proteus vulgaris can be used for the production of (R)-2-hydroxy-4-phenylbutanoic acid [5]. The asymmetric reduction of 2-oxo-4-phenylbutanoic acid ethyl ester to the corresponding (R)-2hydroxy-4-phenylbutanoic acid ethyl ester was likewise investigated by Chadha [35]. The biotransformation procedure was carried out by cell cultures of Daucus carota, producing a high yield and enantiomeric excess of the product. The adopted method obviates the problem of racemate mixture resolution, as only enantiopure product was produced.

\subsection{ACE INHIBITOR DRUG PRECURSOR SYNTHESIS USING MEMBRANE BIOREACTOR WITH POTENTIAL INDUSTRIAL APPLICATION}

Production of ACE inhibitor intermediates has been studied for many years, as discussed in the previous section [12, 19, 22, 25, 30-35], generating substantial literatures and knowledge with respect to the bioreactor systems. Presently, many different types of laboratory bioreactors are used worldwide for the production of ACE inhibitor drug precursors. Conventional laboratory bioreactors require separate and often complicated downstream processing for recovery or retention of isolated enzymes from the aqueous media. Isolated enzyme processes are simple to implement because they are amenable to rapid process development and there are few side reactions. However, the enzymes need to retained and reused in order to keep the operating costs manageable [36]. Membranes provide essential barriers to the biocatalysts, effectively retaining them in soluble form while allowing transport of substrates and products to and from the homogeneous solution [37]. In such circumstances, by exploiting the advantages of combining membranes and biocatalysts, membrane bioreactors with conventional reaction and product separation units [5], have been engineered to produce various ACE inhibitor drug precursors with high selectivity and productivity under mild conditions. Production of the ACE inhibitor intermediates are generally carried out in a continuously operated stirred tank reactor with an additional membrane separation unit to retain the enzymes either in their free soluble state or immobilized onto the membrane.

For the production of (R)-2-hydroxy-4-phenylbutanoic acid as one of the drugs intermediates, one of the successfully published methods involve the usage of D-lactate-NAD oxidoreductase as the solubilized enzyme for the enzymatic reduction 
redox reaction in aqueous solution. A bioreactor equipped with a detached ultrafiltration membrane separation unit is used for production of the precursor; with regeneration of cofactor by a coupled reaction system [38-41]. This system could simultaneously be adopted for the production of the (S)-2-antino-4-phenylbutanoic acid counterpart, using L-homophenylalanine dehydrogenase as the appropriate enzyme [42, 43]. Membrane unit operations usually work under mild conditions and are environmental safe processes [44], as depicted in the synthesis of ACE inhibitor intermediates with low working temperature and pressure, with the additional advantage of minimum diffusional resistance due to direct contact between substrate and biocatalyst [45].

In contrast to this situation, membrane bioreactors are also employed for immobilization of enzymes or whole cells to provide high retention of enzyme activity, high rates of mass transfer, ease of enzyme replacement and for the latter, an environment for increased cell densities to obtain higher product yield. The enzymes or cells are retained in the bioreactor through a membrane barrier, and the membrane bioreactors could be configured in microcapsule, hollow fiber or flat sheet reactor modules, each with their respective pros and cons [46]. The hollow fiber and flat sheet membrane bioreactors could also be applied for the synthesis of ACE inhibitor drug precursors where whole cells could be immobilized on the membrane for better yield.

Although membrane bioreactors have gained widespread application, the development of compact and integrated one-unit-joint-application meliorated bioreactors with efficient enzyme retention system for drug intermediate production has remained by and large uncharted, with ample room for innovation. With respect to this context, enhanced performance bioreactors particularly integrated membrane bioreactors could be utilized for economically competitive and practicable largescale drug precursor production process. This technology has emerged as a result of advances made in the areas of membrane technology for wastewater treatment, but remained largely unexplored in the production of pharmaceutical intermediates. The system usually malkes use of a reactor module, coupled to a separation unit for the continuous removal of products while retaining the biocatalysts. It is projected that the use of integrated membranes in the system would generate a more effective and efficient process. The substrates and the coenzyme NAD could be pumped through a sterile filter into the membrane bioreactor, with the product leaving the membrane bioreactor through an ultrafiltration membrane with varying molecular weight cut off for different enzymes. Enzymes are to be supplemented periodically dependent on the deactivation rates via a sterile filter to the membrane bioreactor. It is of significance to note that this reduction process could be optimized, with room for improvement particularly in the amount of buffer, water and solvent used.

\subsection{CONCLUSION}

The number of possibilities for integrating the membrane bioreactor seems to be vast, bearing in mind that the cost of pharmaceuticals production has become one of the prominent factors for production of single enantiomers. The integrated retrofit system offers numerous advantages including a compact design with small footprint, low system and operating cost, and complete enzyme removal in a single unit. Although ACE inhibitor precursors have been produced industrially, the large scale technology is still at an emerging stage. The key-point to success is the process of transferring the laboratory procedures into cost-effective and reliable plant scale operation, in which the design of integrated membrane bioreactor usurps a significant role, thus sufficient knowledge should be generated for their ultimate development.

\section{ACKNOWLEDGEMENT}

This work was supported by the Malaysian Ministry of Science, Technology and Innovation (MOSTI) under Science Fund Grant No. 6013204 for 2007 2009. Oh P.C. gratefully acknowledges the National Science Fellowship (NSF) received from MOSTI for her Ph.D program. 


\section{REFERENCES}

[1] Liese, A., U. Kragl, H. Kierkels, and B. Schulze. 2002. Membrane Reactor Development for the Kinetic Resolution of Ethyl 2-hydroxy-4-phenylbutyrate. Enzyme and Microbial Technology. 30: 673-681.

[2] Trevor, A.J., B.G. Katzung, and S.B. Masters. 2002. Katzung \& Trevor's Pharmacology. $6^{\text {th }}$ ed. New York: McGraw-Hill Companies, Inc. 662.

[3] Ondetti, M.A., and D.W. Cushman. 1981. Inhibition of the Renin-Angiotensin System: A New Approach to the Therapy of Hypertension. Journal of Medicinal Chemistry. 24: 355-361.

[4] McMurray, J., A. Cohen-Solal, R. Dietz; E: Eichhorn, L. Erhardt, F.D.R. Hobbs, H. Krum, A. Maggioni, RS. McKelvie, I.L. Pina, J. Soler-Soler, and K. Swedberg. 2005. Practical Recommendations for the Use of ACE Inhibitors, Beta-blockers, Aldosterone Antagonists and Angiotensin Receptor Blockers in Heart Failure: Putting Guidelines into Practice. European Journal of Heart Failure 7: 710-721.

[5] Liese, A., K. Seelbach, and C. Wandrey. 2000. Industrial Biotransformationsed. Weinheim: Wiley-VCH Verlag GmbH. 423.

[6] Johnson, A.L., W.A. Price, P.C. Wong, R.F. Vavala, and J.M. Stump. 1985. Synthesis and Pharmacology of the Potent AngiotensinConverting Enzyme-Inhibitor N-[1(S)(Ethoxycarbonyl)-3-Phenylprophyl]-(S)Alanyl-(S)-Pyroglutamic Acid. Journal of Medicinal Chemistry. 28: 1596-1602.

[7] Larissegger-Schnell, B., S.M. Glueck, W. Kroutil, and K. Faber. 2006. EnantioComplementary Deracemization of $(+/-)$ 2-hydroxy-4-phenylbutanoic acid and (+/-)3-phenyllactic Acid Using Lipase-catalyzed Kinetic Resolution Combined with Biocatalytic Racemization. Tetrahedron. 62: 2912-2916.

[8] Herold, P., A.F. Indolese, M. Studer, H.P. Jalett, U. Siegrist, and H.U. Blaser. 2000. New Technical Synthesis of Ethyl (R)-2-hydroxy4-phenylbutyrate of High Enanti-omeric Purity. Tetrahedron. 56: 6497-6499.
[9] Williams, R.M., P.J. Sinclair, D. Zhai, and D. Chen. 1988. Practical Asymmetric Syntheses of Alpha-Amino-Acids through Carbon Carbon Bond Constructions on Electro-philic Glycine Templates. Journal of the American Chemical Society. 110: 15471557.

[10] Ahuja, S. 1997. Chiral Separations: Application and Technologyed. American Chemical Society. 349.

[11] Patel, R.N., A. Goswami, L. Chu, M.J. Donovan, V. Nanduri, S. Goldberg, R. Johnston, P.J. Siva, B. Nielsen, J.Y. Fan, W.X. He, Z.P. Shi, K.W. Wang, R. Eiring; D. Cazzulino, A. Singh, and R. Mueller. 2004. Enantioselective Microbial Reduction of Substituted Acetophenones. TetrahedronAsymmetry. 15: 1247-1258.

[12] de Lacerda, P.S.B., J.B. Ribeiro, S.G.F. Leite, R.B. Coelho, E.L.D. Lima, and O.A.C. Antunes. 2006. Microbial Enantio-selective Reduction of Ethyl-2-oxo-4-phenyl-butanoate. Biochemical Engineering Journal. 28: 299-302.

[13] Ribeiro, J.B., M.D. Ramos, F.R.D. Neto, S.G.F. Leite, and O.A.C. Antunes. 2003. Microbiological Enantioselective Reduction of Ethyl Acetoacetate. Journal of Molecular Catalysis B-Enzymatic. 24-5: 121-124.

[14] Ribeiro, J.B., M.D. Ramos, F.R. Neto, S.G.F. Leite, and O.A.C. Antunes. 2005. New Microbiological Catalytic Accesses to (S)fluoxetine. Catalysis Communications. 6: 131-133.

[15] Roberts, S.M. 1998. Preparative Biotransformations: The Employment of Enzymes and Whole-cells in Synthetic Organic Chemistry. Journal of the Chemical Society-Perkin Transactions 1. 157-169.

[16] Iwasaki, G., R. Kimura, N. Numao, and K. Kondo. 1989. A Practical and Diastereoselective Synthesis of Angiotensin Converting Enzyme-Inhibitors. Chemical \& Pharmaceutical Bulletin. 37: 280-283.

[17] Wang, Y.F, S.T. Chen, K.K.C. Liu, and C.H. Wong. 1989. Lipase-Catalyzed Irreversible Trans-Esterification Using Enol Esters: Resolution of Cyanohydrins and Syntheses of Ethyl (R)-2-Hydroxy-4-Phenylbutyrate 
and (S)-Propranolol. Tetrahedron Letters. 30: 1917-1920.

[18] Sheldon, R.A. 1993. Chirotechnology Industrial Synthesis of Optically Active Compoundsed. New York: Marcel Dekker, Inc. 423.

[19] Chadha, A., and M. Manohar. 1995. Enzymatic Resolution of 2-Hydroxy-4Phenylbutanoic Acid and 2-Hydroxy-4Phenylbutenoic Acid. TetrahedronAsymmetry. 6: 651-652.

[20] Servi, S., D. Tessaro, and G. PedrocchiFantoni. 2008. Chemo-enzymatic Deracemization Methods for the Preparation of Enantiopure Non-natural Alpha-amino Acids. Coordination Chemistry Reviews. 252: 715-726.

[21] Liese, A., and M. Villela. 1999. Production of Fine Chemicals Using Biocatalysis. Current Opinion in Biotechnology. 10: 595603.

[22] Bradshaw, C.W., C.H. Wong, W. Hummel, and M.R. Kula. 1991. Enzyme-Catalyzed Asymmetric-Synthesis of (S)-2-Amino-4Phenylbutanoic Acid and (R)-2-Hydroxy-4Phenylbutanoic Acid. Bioorganic Chemistry. 19: 29-39.

[23] Busca, P., F. Paradisi, E. Moynihan, A.R. Maguire, and P.C. Engel. 2004. Enantioselective Synthesis of Non-Natural Amino Acids Using Phenylalanine Dehydrogenases Modified by Site-Directed Mutagenesis. Organic \& Biomolecular Chemistry. 2 : 2684-2691.

[24] Bommarius, A.S., M. Schwarm, and K. Drauz. 1998. Biocatalysis to Amino AcidBased Chiral Pharmaceuticals - Exámples and Perspectives. Journal of Molecular Catalysis B-Enzymatic. 5: 1-11.

[25] Asano, Y., A. Yamada, Y. Kato, K. Yamaguchi, Y. Hibino, K. Hirai, and K. Kondo. 1990. Enantioselective Synthesis of (S)-Amino Acids by Phenylalanine Dehydrogenase from Bacillus-Sphaericus: Use of Natural and Recombinant Enzymes. Journal of Organic Chemistry. 55: 5567-5571.

[26] Kragl, U., W. Kruse, W. Hummel, and C. Wandrey. 1996. Enzyme Engineering Aspects of Biocatalysis: Cofactor Regene- ration As Example. Biotechnology and Bioengineering. 52: 309-319.

[27] Politino, M., M.M. Cadin, P.M. Skonezny, and J.G. Chen. 2005. Process for Preparing Dipeptidyl IV Inhibitors and Intermediates Therefor. WO 2005/106011 A3.

[28] Hanson, R., M.J. Donovan, S. Goldberg, P.A. Jass, W.S. Li, R. Patel, K. Ramig, L.J. Szarka, and J.J. Venit. 2000. Stereoselective Reductive Amination of Ketones. Patent, U.S. $6,140,088$.

[29] Shaked, Z., and G.M. Whitesides. 1980. Enzyme-Catalyzed Organic-Synthesis NADH Regeneration by Using Formate Dehydrogenase. Journal of the American Chemical Society. 102: 7104-7105.

[30] Chen, S.T., M.J. Tseng, T. Kao, B. Sooklkheo, and T. Surat. 2000. Facile Synthesis of LHomophenylalanine By Equilibrium Shift Enzymatic Reaction Using Engineered Tyrosine Aminotransferase. U.S. Patent $6,146,859$.

[31] Senuma, M., K. Nakamichi, K. Nabe, S. Nishimoto, and T. Tosa. 1989. IndustrialProduction of L-2-Amino-4-Phenylbutyric Acid from 2-Oxo-4-Phenylbutyric Acid by Paracoccus Denitrificans Containing Aminotransferase Activity. Applied Biochemistry and Biotechnology. 22: 141-150.

[32] Cho, B.K., J.H. Seo, T.W. Kang, and B.G. Kim. 2003. Asymmetric Synthesis of L-Homophenylalanine by Equilibrium-Shift Using Recombinant Aromatic L-Amino Acid Transaminase. Biotechnology and Bioengineering. 83: 226-234.

[33] Kao, C.H., H.H. Lo, S.K. Hsu, and W.H. Hsu. 2008. A Novel Hydantoinase Process Using Recombinant Escherichia Coli Cells with Dihydropyrimidinase, and L-N-Carbamoylase Activities As Biocatalyst for the Production of L-homophenylalanine. Journal of Biotechnology. 134: 231-239.

[34] de Lacerda, P.S.B., J.B. Ribeiro, S.G.F. Leite, M.A. Ferrara, R.B. Coelho, E.P.S. Bon, E.L.D. Lima, and O.A.C. Antunes. 2006. Microbial Reduction of Ethyl 2-oxo-4-phenylbutyrate. Searching for R-enantioselectivity. New Access to the Enalapril like ACE Inhibitors. Tetrahedron-Asymmetry. 17: 1186-1188. 
[35] Chadha, A., M. Manohar, T. Soundararajan, and T.S. Lokeswari. 1996. Asymmetric Reduction of 2-Oxo-4-Phenylbutanoic Acid Ethyl Ester by Daucus carota Cell Cultures. Tetrahedron-Asymmetry. 7: 1571-1572.

[36] Pollard, D.J., and J.M. Woodley. 2007. Biocatalysis for Pharmaceutical Intermediates: The Future is Now. Trends in Biotechnology. 25: 66-73.

[37] Wichmann, R., C. Wandrey, A.F. Buckmann, and M.R. Kula. 2000. Continuous Enzymatic Transformation in an Enzyme Membrane Reactor with Simultaneous NAD (H) Regeneration (Reprinted from Biotechnology and Bioengineering, 1981; 23, 2789-2802). Biotechnology and Bioengineering. 67: 791-804.

[38] Schmidt, E., O. Ghisalba, D. Gygax, and G. Sedelmeier. 1992. Optimization of a Process for the Production of (R)-2hydroxy-4-phenylbutyric acid - An Intermediate for Inhibitors of Angiotensin Converting Enzyme. Journal of Biotechnology. 24: 315-327.

[39] Blaser, H.U., M. Eissen, P.F. Fauquex, K. Hungerbuehler, E. Schmidt, G. Sedelmeier, and M. Studer. 2004. Comparison of Four Technical Syntheses of Ethyl (R)-2-hydroxy4-phenylbutyrate.ed. Weinheim: Wiley-VCH Verlag GmbH \& Co. KGaA. 91-103.

[40] Schmidt, E., O. Ghisalba, D. Gygax, and G. Sedelmeier. 1992. Optimization of a Process for the Production of (R)-2-lyydroxy-4phenylbutyric Acid: An Intermediate for Inhibitors of Angiotensin Converting Enzyme. Journal of Biotechnology. 24: 315327.

[41] Liu, W.F., and P. Wang. 2007. Cofactor Regeneration for Sustainable Enzymatic Biosynthesis, Biotechnology Advances. 25: 369-384.

[42] Patel, R.N., 2000. Stereoselective Biocatalysis. New York: Marcel Dekker, Inc. 932.

[43] Krix, G., A.S. Bommarius, K. Drauz, M. Kottenhahn, M. Schwarm, and M.R. Kula. 1997. Enzymatic Reduction of Alpha-keto Acids Leading to L-Amino Acids, D- or LHydroxy Acids. Journal of Biotechnology. 53 : 29-39.

[44] Belafi-Balko, K., L. Gubicza, and M. Mulder, 2000. Integration of Membrane Processes Into Bioconversions. New York: Kluwer Academic/Plenum Publishers. 242.

[45] Fiechter, A., 1983. Advances in Biochemical Engineering/Biotechnology. New York: Springer-Verlag.

[46] Belfort, G., and C.A. Heath. 1993. Enzyme Membrane Reactors. In Proceedings of the NATO Advanced Study Institute on Membrane Processes in Separation and Purification (Crespo, J. G. and Boddeker, K.W., eds.), Kluwer Academic Publishers. 149-168. 